\title{
ALEMTUZUMAB USE IN NEUROMYELITIS OPTICA SPECTRUM DISORDERS - A BRIEF CASE SERIES
}

Laura Azzopardi ${ }^{1}$, Amanda L Cox ${ }^{2}$, Claire L McCarthy ${ }^{2}$, Joanne L Jones ${ }^{1}$, Alasdair J Coles ${ }^{1}$

${ }^{1}$ Department of Clinical Neurosciences, University of Cambridge, Cambridge, UK

${ }^{2}$ Department of Clinical Neurosciences, Addenbrooke's Hospital, Cambridge, UK

Corresponding author: Dr Laura Azzopardi, Department of Clinical Neurosciences, University of

Cambridge, Box 165, Cambridge Biomedical Campus, Cambridge CB2 0QQ.

laura.azzopardi@gmail.com, Tel: (+44) 01223 762051, Fax: (+44) 01223769318.

Keywords: Alemtuzumab, Neuromyelitis Optica, Multiple Sclerosis, Neuroimmunology

\section{Acknowledgements}

Clinical work is performed at the Wellcome Clinical Research Facility. JL and AJC are supported by the Cambridge Biomedical Research Centre of the National Institute for Health Research.

Number of figures: 1

Number of tables: 1 
Azzopardi et al: Alemtuzumab use in Neuromyelitis Optica Spectrum Disorders - a brief case series

\section{ABSTRACT}

Alemtuzumab is an anti-CD52 monoclonal antibody recently licensed for use in relapsing-remitting multiple sclerosis. Here we report our experience of its use in neuromyelitis optica (NMO) spectrum disorders.

A retrospective case review of patients treated with alemtuzumab in Cambridge, UK, was conducted to identify those who fulfil the criteria for NMO spectrum disorder. Three cases were identified. Case 1, 9-year old female, presented with transverse myelitis and bilateral optic neuritis, with one lower medullary and several longitudinally extensive cord lesions. Despite immunosuppression including two courses of alemtuzumab, she continued to relapse, was wheelchair bound and registered blind by age 12 , and died at age 18 . Case 2, 41-year old female, presented with bilateral optic neuritis and transverse myelitis with longitudinally extensive cervical cord lesions. Despite three courses of alemtuzumab, she had 5 relapses with visual impairment and new cord lesions. She later developed tumefactive white matter lesions and died aged 51. Case 3, 31-year old female, presented with transverse myelitis with longitudinally extensive cervical cord lesions and positive aquaporin-4 antibody. After one course of alemtuzumab, she relapsed with 4 episodes of myelitis with new enhancing lesions and accumulating disability. She became relapse-free after rituximab and mycophenolate mofetil.

From this case series we conclude that alemtuzumab failed to prevent disabling relapses and poor outcome in NMO. We Case 3 stabilised with rituximabhypothesise that rituximab is more effective, as in Case 3, because it causes much more, the important mechanistic difference being prolonged $B$ lymphocyte depletion, which is only depleted for 1-2 months after than alemtuzumab. We therefore caution against the use of alemtuzumab in NMO. 
Azzopardi et al: Alemtuzumab use in Neuromyelitis Optica Spectrum Disorders - a brief case series

\section{MANUSCRIPT TEXT}

\section{Introduction}

Alemtuzumab is an anti-CD52 humanised monoclonal antibody recently approved in the European Union and by the U.S. Food and Drug Administration as a therapeutic agent in relapsing-remitting multiple sclerosis (RRMS). Alemtuzumab induces a profound lymphopaenia; reconstitution of the various lymphocyte subsets leads to prolonged modulation of the immune repertoire [1-3]. This leads to significant reductions in both relapse frequency and sustained accumulation of disability, compared to interferon beta-1a, in RRMS [4-6].

Neuromyelitis optica (NMO) spectrum disorders are a group of syndromes caused by antibodies, discovered in 2004 [7], targeting the aquaporin-4 water channel on astrocytic foot processes [8]. We and others have occasionally used alemtuzumab to treat NMO, both before and after NMO was recognised as a distinct clinical entity, and here report our experience.

\section{Methods}

We conducted a retrospective case review, from 1991 to the present day, of patients treated with alemtuzumab at the Department of Neurology in Addenbrooke's Hospital, Cambridge, UK, to identify those patients who fulfil the criteria for NMO spectrum disorder under the revised Wingerchuk criteria [9] and the consensus criteria drawn up by the International Panel for NMO Diagnosis convened in 2011 [10]. The main outcome measures considered were relapse rate, accumulation of disability and long-term outcome after alemtuzumab treatment. 
Azzopardi et al: Alemtuzumab use in Neuromyelitis Optica Spectrum Disorders - a brief case series

\section{Results}

In retrospect, three cases could be classified as neuromyelitis optica. One case (case 1) was from the 1990s; the remaining two were from the early 2000s.

\section{Case 1}

A 9-year old girl presented in 1998 with protracted vomiting followed by transverse myelitis, two relapses of bilateral optic neuritis and two brainstem episodes (nystagmus, ataxia and facial sensory disturbance) within a year. Imaging showed one lower medullary and several longitudinally extensive cord lesions (images no longer available). Despite high-dose steroids and intensive immunosuppression (Table 1), she remained significantly visually impaired (acuities down to 6/60), as well as having a spastic paraparesis. She received alemtuzumab with no relapses for twelve months. At month 12 she relapsed with myelitis with longitudinally-extensive thoracic cord demyelination and was retreated with alemtuzumab; only weeks later a further relapse resulted in dense paraparesis, rendering her wheel-chair bound. Her vision deteriorated further and she was registered blind by age 12. In 2005, her illness was recognised as antibody-mediated NMO [7] and she received plasma exchange (PLEX). She died at age 18, whilst awaiting rituximab treatment. Assays for aquaporin-4 immunoglobulin-G (AQP4-IgG) were not available at the time.

\section{Case 2}

A 41-year old female presented in 2004 with bilateral optic neuritis and a brainstem deficit (left-sided horizontal nystagmus and ataxia) and 2 episodes of myelitis within six months; imaging showed enhancing brain and longitudinally-extensive cervical cord lesions (Fig 1a-c). She received pulsed highdose steroids and remained with residual spastic paraparesis. Three cycles of alemtuzumab were 
Azzopardi et al: Alemtuzumab use in Neuromyelitis Optica Spectrum Disorders - a brief case series administered 12 months apart. At months 3, 4 and 11 after the first cycle she had discrete relapses attributable to new thoracic cord lesions (Fig 1d), whilst cerebral lesions improved (not shown). At months 2 and 9 after second cycle, she relapsed with brainstem symptoms (vertigo and incoordination) and bilateral optic neuritis, with residual visual impairment (6/12). After the third cycle she deteriorated with disabling cognitive deficits. She received rituximab, to no effect, later developing seizures and tumefactive white matter lesions (Fig 1e, 1f). A brain biopsy performed to confirm active demyelination showed extensive loss of myelin with numerous $\mathrm{CD}^{+} 8^{+}$macrophages throughout, but the specific features of neuromyelitis optica were not sought. She went on to be treated with mitoxantrone with poor response. Late in the course of her illness, as the assay became available, she was tested for aquaporin-4 immungglobulin-G (AQP4-IgG t antibodies, which were absent. She died aged 51.

Case 3

A 31-year old female presented in 2007 with two episodes of transverse myelitis with longitudinally extensive cervical cord lesions (Fig 1g, 1h) and tested seropositive for AQP4-IgG antibody. Two years prior, she had a prolonged episode of nausea, vomiting and diplopia that had resolved spontaneously. Response to high-dose steroids and PLEX was moderate, with residual paraesthesia, unsteadiness and tonic spasms. She received a single course of alemtuzumab (12mg daily for 5 consecutive days) in an attempt to prevent further relapses but at months 3 and 5 after alemtuzumab she represented with spastic paraparesis; cord imaging showed disease progression with new enhancing longitudinallyextensive spinal cord lesions (Fig $1 \mathrm{i}, 1 \mathrm{j}$ ). At months 10 and 18 she had further relapses of myelitis without cord enhancement, losing dominant hand function and ability to walk unassisted despite highdose steroids and PLEX. She became relapse-free for 4 years after two cycles of rituximab in combination with mycophenolate mofetil (MMF), though disability persists with cord atrophy (Fig $1 \mathrm{k}$ ). Serum AQP4-IgG antibody remained positive after alemtuzumab treatment. 
Azzopardi et al: Alemtuzumab use in Neuromyelitis Optica Spectrum Disorders - a brief case series

\section{Discussion}

We report three cases which demonstrate that alemtuzumab failed to prevent disabling relapses and

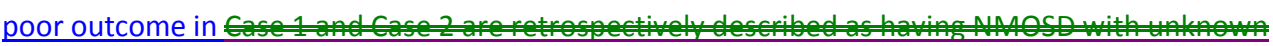

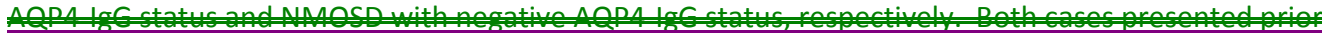

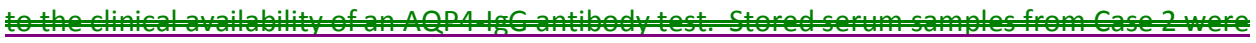

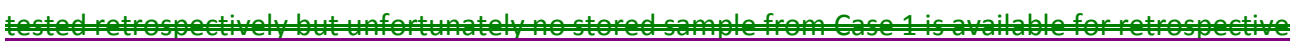

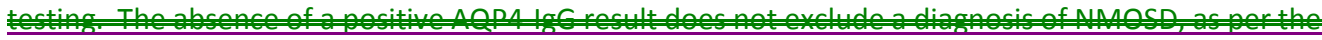
International consensus diagnostic criteria draw u 2011 [10]. We feel that both patients would

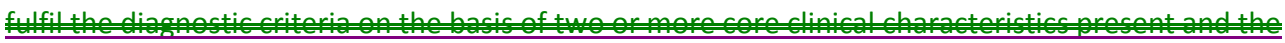
ex of a

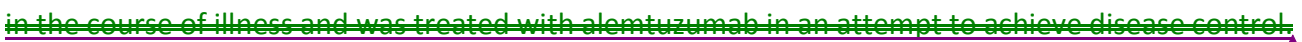

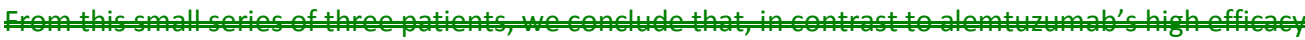

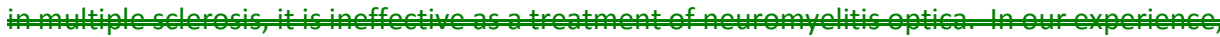

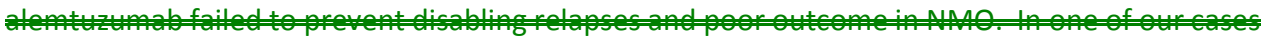

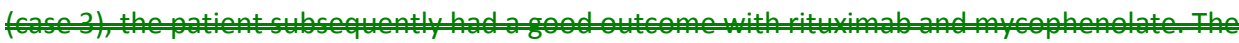

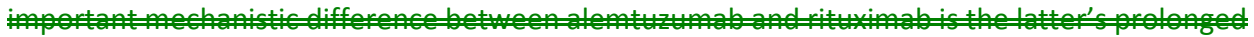

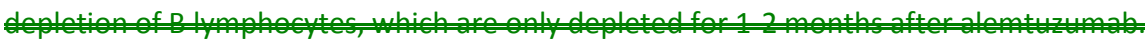

Although we report the experience from only three cases, the failure of alemtuzumab to control neuromyelitis optica. This converges from evidence from other drugs effective in multiple sclerosis which also fail to suppress - and indeed may exacerbate - the illness, like interferon beta and natalizumab $[11,12]$. We are aware of two other cases of neuromyelitis optica treated using alemtuzumab. In one, there was no effect on the disease which was subsequently effectively treated using an IL-6 antibody [13]. In another, there was a cessation of relapses and reduction in 
Azzopardi et al: Alemtuzumab use in Neuromyelitis Optica Spectrum Disorders - a brief case series lymphocytes detected in brain lesions pathologically, but the patient deteriorated and died with significant monocyte invasion of her brain lesions [14].

All three cases fulfil the criteria for NMO spectrum disorder under the revised Wingerchuk criteria [9] and the International Panel for NMO Diagnosis consesnsus [10]. The authors interpret this as a demonstration of the pathogenicity of the innate immune response in neuromyelitis optica, even When alemtuzumab had successfully suppressed adaptive immune mechanisms.Case 1 and Case 2 are retrospectively described as having NMOSD with unknown AQP4-IgG status and NMOSD with negative AQP4-IgG-status, respectively. Both cases 1 and 2 presented prior to the clinical availability of an AQP4-IgG antibody test; $;$ Sstored serum samples from Case 2 were testednegative; case 3 was positive in life. retrospectively but unfortunately no stored sample from Case 1 is a vailable for retrospective testing: The absence of a positive AQP4-IgG result does not exclude a diagnosis of NAOSD, as per the International consensus diagnostic criteria drawn up in 2011] [10]. We feel that both patients would fulfil the diagnostic criteria on the basis of two or more core clinical characteristics present and the exclusion of alternative diagnoses. Case 3 was recognised as NMOSD with AQP4-IgG antibody early on in the course of illness and was treated with alemtuzumab in an attempt to achieve disease control. From this small series of three patients, we conclude that, in contrast to alemtuzumab's high efficacy in multiple sclerosis, it is ineffective as a treatment of neuromyelitis optica. In our experience, alemtuzumab failed to prevent disabling relapses and poor outcome in NAMO. In one of our cases (case 3), the patient subsequently had a good outcome with rituximab and mycophenolate. We hypothesise that the The-important mechanistic difference between alemtuzumab and rituximab is the latter's prolonged depletion of B lymphocytes, which are only depleted for 1-2 months after alemtuzumab. The use of alemtuzumab is associated with secondary autoimmunity, directed mainly against the thyroid, but we do not propose this mechanism for continued disease activity of NMO after alemtuzumab. 
Azzopardi et al: Alemtuzumab use in Neuromyelitis Optica Spectrum Disorders - a brief case series

From this case series, and a review of the literature, we therefore caution against the use of alemtuzumab in cases of neuromyelitis optica. 
Azzopardi et al: Alemtuzumab use in Neuromyelitis Optica Spectrum Disorders - a brief case series

\section{Conflict of Interest}

$\mathrm{JU}$ reports receiving consulting fees and lecture fees from Genzyme Sanofi. AJC reports receiving consulting fees, lecture fees and institutional grant support from Genzyme Sanofi. LA, ALC and CMC declare they have no conflict of interest.

\section{Ethical standards}

All patients provided informed consent to alemtuzumab treatment and long-term follow-up at Addenbrooke's Hospital, Cambridge, UK. 
Azzopardi et al: Alemtuzumab use in Neuromyelitis Optica Spectrum Disorders - a brief case series

\section{References}

1. Cox AL, Thompson SA, Jones JL, Robertson VH, Hale G, Waldmann H, Compston DA, Coles AJ (2005) Lymphocyte homeostasis following therapeutic lymphocyte depletion in multiple sclerosis. Eur $J$ Immunol 35 (11):3332-3342. doi:10.1002/eji.200535075

2. Jones JL, Thompson SAJ, Loh P, Davies JL, Tuohy OC, Curry AJ, Azzopardi L, Hill-Cawthorne G Fahey MT, Compston A, Coles AJ (2013) Human autoimmunity after lymphocyte depletion is caused by homeostatic T-cell proliferation. Proceedings of the National Academy of Sciences.

doi:10.1073/pnas.1313654110

3. Hill-Cawthorne GA, Button T, Tuohy O, Jones JL, May K, Somerfield J, Green A, Giovannoni G, Compston DA, Fahey MT, Coles AJ (2012) Long term lymphocyte reconstitution after alemtuzumab treatment of multiple sclerosis. Journal of neurology, neurosurgery, and psychiatry 83 (3):298-304. doi:10.1136/jnnp-2011-300826

4. Coles AJ, Fox E, Vladic A, Gazda SK, Brinar V, Selmaj KW, Skoromets A, Stolyarov I, Bass A Sullivan H, Margolin DH, Lake SL, Moran S, Palmer J, Smith MS, Compston DA (2012) Alemtuzumab more effective than interferon beta-1a at 5-year follow-up of CAMMS223 clinical trial. Neurology 78 (14):1069-1078. doi:10.1212/WNL.0b013e31824e8ee7

5. Cohen JA, Coles AJ, Arnold DL, Confavreux C, Fox EJ, Hartung H-P, Havrdova E, Selmaj KW, Weiner HL, Fisher E, Brinar VV, Giovannoni G, Stojanovic M, Ertik BI, Lake SL, Margolin DH, Panzara MA, Compston DAS (2012) Alemtuzumab versus interferon beta 1a as first-line treatment for patients with relapsing-remitting multiple sclerosis: a randomised controlled phase 3 trial. The Lancet 6. Coles AJ, Twyman CL, Arnold DL, Cohen JA, Confavreux C, Fox EJ, Hartung HP, Havrdova E, Selmaj KW, Weiner HL, Miller T, Fisher E, Sandbrink R, Lake SL, Margolin DH, Oyuela P, Panzara MA, Compston DA (2012) Alemtuzumab for patients with relapsing multiple sclerosis after disease-modifying therapy: a randomised controlled phase 3 trial. Lancet 380 (9856):1829-1839. doi:10.1016/s01406736(12)61768-1

7. Lennon VA, Wingerchuk DM, Kryzer TJ, Pittock SJ, Lucchinetti CF, Fujihara K, Nakashima I, Weinshenker BG (2004) A serum autoantibody marker of neuromyelitis optica: distinction from multiple sclerosis. Lancet 364 (9451):2106-2112. doi:10.1016/s0140-6736(04)17551-x

8. Lucchinetti CF, Guo Y, Popescu BF, Fujihara K, Itoyama Y, Misu T (2014) The pathology of an autoimmune astrocytopathy: lessons learned from neuromyelitis optica. Brain pathology (Zurich, Switzerland) 24 (1):83-97. doi:10.1111/bpa.12099

9. Wingerchuk DM, Lennon VA, Pittock SJ, Lucchinetti CF, Weinshenker BG (2006) Revised diagnostic criteria for neuromyelitis optica. Neurology 66 (10):1485-1489.

doi:10.1212/01.wnl.0000216139.44259.74

10. Wingerchuk D, Banwell B, Bennett J, Cabre P, Carroll W, Chitnis T, De Seze J, Fujihara K, Greenberg B, Jacob A, Jarius S, Lana-Peixoto M, Levy M, Simon J, Tenembaum S, Traboulsee A, Waters P, Wellik K, Weinshenker B (2014) Revised Diagnostic Criteria for Neuromyelitis Optica Spectrum Disorders (S63.001). Neurology 82 (10 Supplement):S63.001

11. Wang KC, Lin KH, Lee TC, Lee CL, Chen SY, Chen SJ, Chin LT, Tsai CP (2014) Poor responses to interferon-beta treatment in patients with neuromyelitis optica and multiple sclerosis with long spinal cord lesions. PLoS One 9 (6):e98192. doi:10.1371/journal.pone.0098192

12. Kleiter I, Hellwig K, Berthele A, Kumpfel T, Linker RA, Hartung HP, Paul F, Aktas O (2012) Failure of natalizumab to prevent relapses in neuromyelitis optica. Archives of neurology 69 (2):239-245. doi:10.1001/archneurol.2011.216

13. Kieseier BC, Stuve O, Dehmel T, Goebels N, Leussink VI, Mausberg AK, Ringelstein M, Turowski B, Aktas O, Antoch G, Hartung HP (2013) Disease amelioration with tocilizumab in a treatment-resistant patient with neuromyelitis optica: implication for cellular immune responses. JAMA neurology 70 (3):390-393. doi:10.1001/jamaneurol.2013.668

14. Gelfand JM, Cotter J, Klingman J, Huang EJ, Cree BA (2014) Massive CNS monocytic infiltration at autopsy in an alemtuzumab-treated patient with NMO. Neurology $(\mathrm{R})$ neuroimmunology \& neuroinflammation 1 (3):e34. doi:10.1212/nxi.0000000000000034 
Azzopardi et al: Alemtuzumab use in Neuromyelitis Optica Spectrum Disorders - a brief case series

\section{Figure legend}

\section{Fig. 1 MRI images}

Case 2 (a) Pre-treatment T2 weighted axial views show high signal in posterior part of corpus callosum and left middle cerebellar peduncle. (b) T2 weighted sagittal view of cervical cord shows expansion with an extensive lesion from medulla to T1 level, and (c) gadolinium enhancement on T1 weighted image. (d) Post-treatment T2 weighted sagittal view shows new expansion of thoracic cord between T4 and T7with increased T2 signal but no gadolinium enhancement. (e) T2 weighted axial images of brain (left) and FLAIR images (right) at Month 20 after third dose of alemtuzumab show a large lesion in right parietal and posterior frontal white matter that extends to posterior corpus callosum and a smaller lesion in left parietal white matter. (f) Both lesions show an incomplete peripheral rim of enhancement on T1 weighted post-gadolinium axial image (left) and coronal image (right). Case 3 (g) Pre-treatment T2 weighted sagittal MRI sequence (left) show subtle expansion of cervical cord from $\mathrm{C} 2$ to $\mathrm{C} 6$ inclusive with diffuse increase in signal, more localised at level of $\mathrm{C} 3 / 4$ as demonstrated on axial view (right). (h) T1 weighed sagittal and axial sequences with gadolinium contrast show enhancement involving the left side of the cord. Worsening of disease at month 5 after alemtuzumab is shown by (i) T2 weighted sagittal views of spine showing progression of $\mathrm{T} 2$ signal change and swelling in cervical cord (left) and new thoracic hyperintensity extending from T2 to T7 (right); with gadolinium enhancement (j). (k) T2 weighted sagittal view of cord shows patchy increased intensity and evidence of cord atrophy, 7 years after disease onset. 